\title{
Cutaneous adverse drug reactions in patients with peripheral blood eosinophilia during antitubercu- losis treatment
}

\author{
Tae-Ok Kim, Hong-Joon Shin ${ }^{*}$ Yu-Il Kim, Sung-Chul Lim, Young-Il Koh, and Yong-Soo Kwon
}

Department of Internal Medicine, Chonnam National University Hospital, Gwangju, Korea

\author{
Received: February 17, 2018 \\ Revised : June 7, 2018 \\ Accepted: July 25, 2018

\section{Correspondence to} \\ Yong-Soo Kwon, M.D. \\ Department of Internal Medi- \\ cine, Chonnam National Uni- \\ versity Hospital, 42 Jebong-ro, \\ Dong-gu, Gwangju 61469, Korea \\ Tel: +82-62-220-6575 \\ Fax: +82-62-225-8578 \\ E-mail:yskwon@jnu.ac.kr
}

*These authors contributed equally to this work.
Background/Aims: Peripheral eosinophilia during tuberculosis (TB) treatment is common, but has not been fully evaluated. The aim of this study was to determine the prevalence and clinical significance of peripheral blood eosinophilia in patients undergoing anti-TB treatment.

Methods: We retrospectively reviewed the clinical and laboratory data of patients who received anti-TB treatment and had peripheral blood eosinophilia ( $>5 \%$ of the total white blood cell count) at the Chonnam National University Hospital between January 2010 and December 2014.

Results: Of all 2,234 patients with TB who received anti-TB treatment, 397 (17.8\%) had peripheral blood eosinophilia. Of the 397 with eosinophilia, we reviewed the data of $262(66 \%)$, and cutaneous adverse drug reactions (CADRs) were observed in $161(61.5 \%)$. Of the 161 with CADRs, itching (47.2\%) and skin rash (47.8\%) were common. Older age, abnormal liver function, and higher peak blood eosinophil percentage were associated with CADRs in multivariate analysis. There was a significant relationship between increased peak eosinophil counts and the degree of severity of CADRs.

Conclusions: Peripheral blood eosinophilia is a relatively common occurrence during anti-TB treatment. Peripheral blood eosinophil counts were higher according to the degree of severity of CADRs.

Keywords: Drug-related side effects and adverse reactions; Antitubercular agents; Drug eruptions; Eosinophilia

\section{INTRODUCTION}

Standard chemotherapy for tuberculosis (TB) is highly effective, but a long duration of treatment and multiple drugs may be required. Therefore, adverse drug reactions (ADRs) during anti-TB treatment are common and can limit their use $[1,2]$. Importantly, ADRs can lead to discontinuation of anti-TB treatment and worse outcomes [3]. Cutaneous adverse drug reactions (CADRs) are among the most common ADRs and a common cause of discontinuation of anti-TB drugs [4]. They can cause reactions that range from a mild presentation with pruritus and a skin rash to fatal and life-threatening hypersensitivity reactions such as Stevens-Johnson syndrome/toxic epidermal necrolysis (SJS/TEN) and the drug reaction with eosinophilia and systemic symptoms (DRESS) syndrome [1,5-13].

Hypersensitivity reactions can be accompanied by peripheral blood eosinophilia $[14,15]$. Antibiotics are an important cause of eosinophilia, and patients with eo- 
sinophilia have a higher risk of skin rash [16]. However, limited information is available regarding peripheral blood eosinophilia during anti-TB treatment. We aimed to evaluate the prevalence and clinical significance of peripheral blood eosinophilia in patients undergoing anti-TB treatment.

\section{METHODS}

\section{Patients and data collection}

We retrospectively reviewed the data of all patients who received treatment for active TB at the Chonnam National University Hospital between January 2010 and December 2014. Patients with peripheral blood eosinophilia during anti-TB treatment were included in this study. Patients transferred to another institution before treatment completion, those with peripheral blood eosinophilia before anti-TB treatment, those lost to follow-up, and those started on anti-TB drugs in other hospitals were excluded.

TB was diagnosed based on Mycobacterium tuberculosis identification with culture or polymerase chain reaction from clinical specimens, or on clinical, radiological, or histological findings compatible with TB and responses to anti-TB treatment. In Korea, a 6-month regimen consisting of a 2-month initial phase of isoniazid, rifampicin, pyrazinamide, and ethambutol, followed by a 4-month continuation phase of isoniazid, rifampicin, and ethambutol has been recommended by the National Tuberculosis Program [17]. Alternatively, a 9-month regimen with isoniazid, rifampin, and ethambutol can be administered. Therefore, all patients in this study received daily therapy consisting of isoniazid (300 $\mathrm{mg}$ ), rifampin ( 450 to $600 \mathrm{mg}$ ), and ethambutol (800 to 1,200 $\mathrm{mg})$, with or without pyrazinamide $(1,500 \mathrm{mg})$. However, the decision to include pyrazinamide in the initial regimen was made by clinicians based on each patient's clinical situation. All patients with T'B in our hospital were registered in a TB registry and received health education as well as monitoring for drug compliance and ADRs during treatment by specially trained nurses who participate in the Public-Private Mix project for TB control in Korea [18]. Anti-TB drugs were self-administered with the support of trained nurses. At each clinic visit, treatment adherence was assessed by asking patients about drug intake and checking the tablets remaining from the previously prescribed regimen. In our hospital, all patients with TB underwent laboratory examinations, including complete blood cell count, liver function tests, renal function tests, and C-reactive protein, before treatment and at every hospital visit, which was scheduled every 1 to 2 months during treatment; if patients had symptoms related to ADRs, including fatigue, rash, and fever, additional laboratory examinations were performed.

The protocol of this retrospective observational study was approved by the Chonnam National University Hospital Institutional Review Board, and the requirement for informed consent was waived because of the retrospective nature of this study (IRB number, CNUH 2018-160).

\section{Eosinophilia and adverse drug reactions}

Eosinophilia was defined as blood eosinophil count above $5 \%$ of total white blood cell count [19]. During a period of eosinophilia, initial peripheral counts and percentages, defined as eosinophil counts and percentages at first detection of eosinophilia, and peak eosinophil counts and percentages, defined as the highest eosinophil counts and percentages, were analyzed to evaluate the differences between patients with and without CADRs.

CADRs were defined as newly developed skin reactions including pruritus, skin rash, DRESS, and SJS/TEN during anti-TB treatment that were accompanied by peripheral blood eosinophilia. SJS/TEN was defined as epidermal detachment and mucous membrane erosion [20]. Patients with CADRs were divided into four groups based on the severity of CADRs. Group 1 had no CADRs, group 2 had itching without skin rash, group 3 had skin rash with or without itching, and group 4 had DRESS syndrome, SJS/TEN, and an uncontrolled skin rash and/ or fever requiring discontinuation of anti-TB drugs.

Abnormal liver function was defined as elevated serum aminotransferase levels more than two times the upper normal limit that were accompanied by peripheral blood eosinophilia [21]. Abnormal renal function was defined as elevated serum creatinine levels more than 1.5 times baseline that were accompanied by peripheral blood eosinophilia [22].

An offending drug was identified when symptoms 


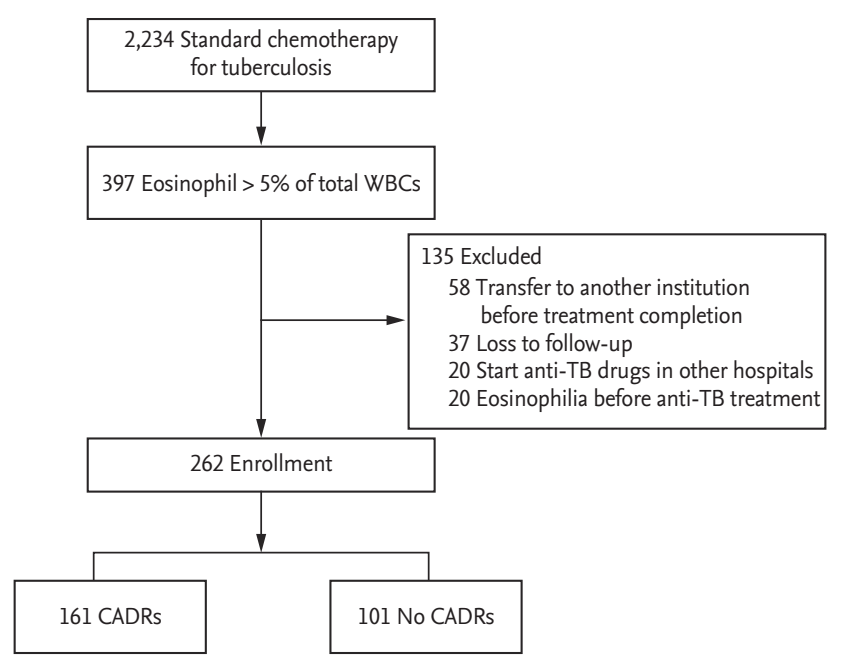

Figure 1. Flow diagram of cutaneous adverse drug reactions (CADRs) associated with peripheral blood eosinophilia during anti-tuberculosis (TB) treatment. WBC, white blood cell.

and signs resolved after drug withdrawal and recurred after rechallenge with that drug.

\section{Statistical analysis}

All data are expressed as medians and interquartile range (IQR) or as numbers (percentages) in the text and tables. Intergroup comparisons were performed using the Mann-Whitney U test for continuous variables, and Pearson's chi-square test or Fisher's exact test for categorical variables. We evaluated trends using the Jonckheere-Terpstra test. Bonferroni tests were used for post hoc correction to account for multiple comparisons. Variables that were statistically significant in univariate analysis were included in multivariable binary logistic regression analysis to determine predictors for CADRs. The level of significance was set at $p<0.05$. Data were analyzed using SPSS for Windows version 22.0 (IBM Co., Armonk, NY, USA).

\section{RESULTS}

\section{Patient characteristics}

Of 2,234 patients who received standard anti-TB treatment during the study period, 397 (17.8\%) had peripheral blood eosinophilia during treatment. Of 397 with peripheral blood eosinophilia, the following were exclud- ed: those transferred to another institution before treatment completion ( $\mathrm{n}=58$ ), those with peripheral blood eosinophilia before starting anti-TB drugs $(n=20)$, those lost to follow-up $(\mathrm{n}=37)$, and those started on anti-TB drugs in other hospitals $(n=20)$. Finally, we enrolled 262 patients with peripheral blood eosinophilia and evaluated the clinical and laboratory findings (Fig. 1).

The median patient age was 60 years (IQR, 45 to 73 ), and $66.8 \%$ were men. CADRs were present in 161 (61.5\%) of 262 patients with peripheral blood eosinophilia. The median blood eosinophil counts and percentage before start of anti-TB treatment were $110 / \mathrm{mm}^{3}$ (IQR, 70 to 200) and $1.9 \%$ (IQR, 0.9 to 3.1), respectively; there was no difference in median blood eosinophil counts between patients with and without CADRs (100 vs. 130, $p=0.177$ ) or in the median blood eosinophil percentage (1.7 vs. 2.1, $p$ = 0.429). Among 161 patients with CADRs, skin rash was the most common presentation, followed by pruritus. The median blood eosinophil counts and percentage at start of CADRs were $400 / \mathrm{mm}^{3}$ (IQR, 300 to 500 ) and $6.2 \%$ (IQR, 5.4 to 7.7), respectively. The peak blood eosinophil count and percentage were $465 / \mathrm{mm}^{3}$ (IQR, 300 to 650 ) and $7.7 \%$ (IQR, 6.0 to 10.7), respectively (Table 1). More than half of the patients (53.1\%) developed peripheral blood eosinophilia within 4 weeks after anti-TB treatment (Fig. 2). Ninety-five patients (36.3\%) had CADRs associated with eosinophilia within 4 weeks. Patients with peripheral blood eosinophilia within 4 weeks had a higher rate of CADRs than patients with eosinophilia after 4 weeks $(68.3 \%$ vs. $54.5 \%, p=0.022)$.

\section{Factors associated with CADRs and comparison of clinical and laboratory findings according to the severity of CADRs in patients with eosinophilia} In multivariate analysis, older age, abnormal liver function, and higher peak blood eosinophil percentage were associated with CADRs (Table 2). The initial and peak blood eosinophil counts and eosinophil percentages tended to increase with the degree of clinical severity of CADRs caused by the anti-TB drug (Jonckheere-Terpstra test, $p<0.001$ ) (Table 3 ).

\section{Offending drugs and reasons for discontinuation}

Twenty-seven (10.3\%) of 262 patients did not continue anti-TB drugs. The most common reasons for discontinuation were drug rash (44.4\%) and drug fever (25.9\%), 
Table 1. Baseline characteristics of patients with eosinophilia during anti-tuberculosis treatment

\begin{tabular}{|c|c|}
\hline Variable & $\operatorname{All}(\mathrm{n}=262)$ \\
\hline Age, yr & $60(45-73)$ \\
\hline Male sex & $175(66.8)$ \\
\hline \multicolumn{2}{|l|}{ Type of TB } \\
\hline Pulmonary TB & $162(61.8)$ \\
\hline Extrapulmonary TB & $100(38.2)$ \\
\hline \multicolumn{2}{|l|}{ Adverse reactions } \\
\hline GI disorder & $118(45.0)$ \\
\hline Abnormal liver function & $32(12.2)$ \\
\hline Abnormal renal function & $10(3.8)$ \\
\hline CADRs & $161(61.5)$ \\
\hline Pruritus only & $76(47.2)$ \\
\hline Skin rash & $77(47.8)$ \\
\hline DRESS & $7(4.4)$ \\
\hline SJS/TEN & $1(0.6)$ \\
\hline Fever & $15(5 \cdot 7)$ \\
\hline \multicolumn{2}{|l|}{ History of previous anti-TB treatment } \\
\hline New treatment & $238(90.8)$ \\
\hline Retreatment & $24(9.2)$ \\
\hline \multicolumn{2}{|l|}{ Peripheral blood eosinophilia } \\
\hline $\begin{array}{l}\text { Eosinophil count before start of } \\
\text { anti-TB treatment, /mL }\end{array}$ & $110(70-200)$ \\
\hline $\begin{array}{l}\text { Eosinophil percentage before start of } \\
\text { anti-TB treatment, \% of total white } \\
\text { blood cell count }\end{array}$ & $1.9(0.9-3.1)$ \\
\hline Initial eosinophil count/mL & $400(300-500)$ \\
\hline $\begin{array}{l}\text { Initial eosinophil percentage, } \% \text { of } \\
\text { total white blood cell count }\end{array}$ & $6.2(5.4-7.7)$ \\
\hline Peak eosinophil count/mL & $465(300-650)$ \\
\hline $\begin{array}{l}\text { Peak eosinophil percentage, \% of total } \\
\text { white blood cell count }\end{array}$ & $7.7(6.0-10.7)$ \\
\hline
\end{tabular}

Values are presented as median (interquartile range) or number (\%).

TB, tuberculosis; GI, gastrointestinal; CADR, cutaneous adverse drug reaction; DRESS, drug reaction with eosinophilia and systemic symptom; SJS/TEN, Stevens-Johnson syndrome/ toxic epidermal necrolysis.

followed by DRESS (25.9\%). The most common offending drug was ethambutol, followed by pyrazinamide and rifampin (Table 4).

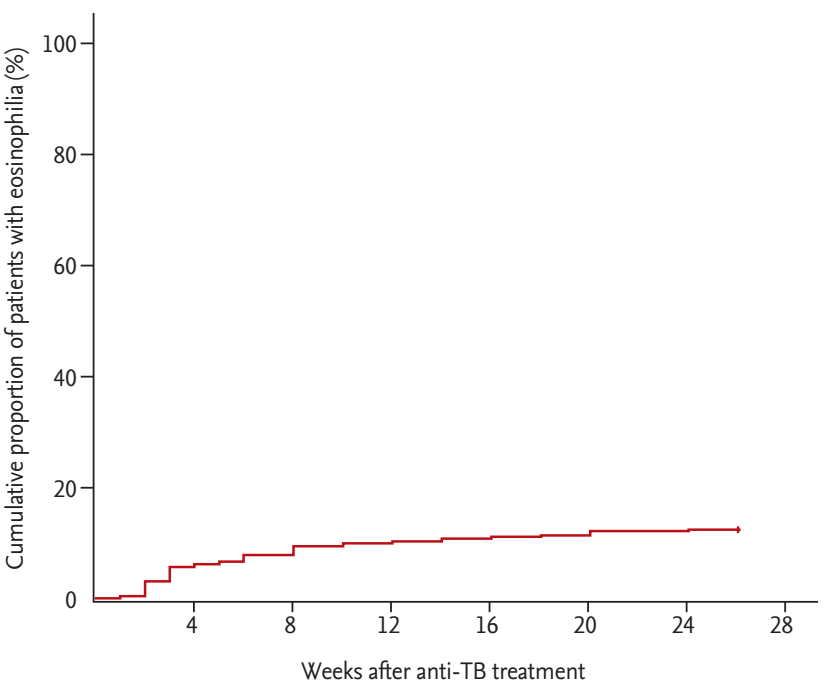

Figure 2. Kaplan-Meier curve for time to development of peripheral blood eosinophilia in patients on anti-tuberculosis (TB) treatment.

\section{DISCUSSION}

In this cohort study, patients with peripheral blood eosinophilia during anti-TB treatment frequently developed CADRs, and the levels of eosinophilia increased according to the severity of the CADRs.

Peripheral blood eosinophilia can be caused by allergic, infectious, and neoplastic disorders. Medications are the most common cause of peripheral blood eosinophilia in developed countries [15]. In our study, a significant number of patients (17.8\%) receiving anti-TB drugs showed peripheral blood eosinophilia, and many (61.5\%) had cutaneous symptoms. This is consistent with a previous study in which the incidence of peripheral blood eosinophilia among patients receiving outpatient parenteral antibiotics was $25 \%$ [16]. However, the incidence of cutaneous symptoms among patients with peripheral blood eosinophilia was higher in our study than in a previous study (61.5\% vs. 15\%) [16]. Although there was no clear cause that could explain the difference, different drugs and duration of treatment might contribute.

It has been reported that eosinophils could be involved in CADRs, according to in vivo and in vitro studies [23-25]. Additionally, CADRs associated with peripheral blood eosinophilia have been reported, especially in DRESS syndrome [1,2,24,26]. Yang et al. [27] reported that CADRs in patients with peripheral blood eosino- 
Table 2. Factors associated with cutaneous adverse drug reactions in patients with eosinophilia during anti-tuberculosis treatment

\begin{tabular}{|c|c|c|c|c|c|}
\hline \multirow{2}{*}{ Variable } & \multirow{2}{*}{$\begin{array}{l}\text { No CADRs } \\
(\mathrm{n}=101)\end{array}$} & \multirow{2}{*}{$\begin{array}{l}\text { CADRs } \\
(\mathrm{n}=161)\end{array}$} & \multirow{2}{*}{$\begin{array}{l}\text { Univariate } \\
\text { p value }\end{array}$} & \multicolumn{2}{|c|}{ Multivariate } \\
\hline & & & & OR $(95 \% \mathrm{CI})$ & $p$ value \\
\hline Age, yr & $56(39-71)$ & $63(46-75)$ & 0.012 & $1.016(1.000-1.032)$ & 0.045 \\
\hline Male sex & $66(65 \cdot 3)$ & $109(67.7)$ & 0.694 & & \\
\hline Type of TB & & & 0.367 & & \\
\hline Pulmonary TB & $59(58.4)$ & $103(64.0)$ & & & \\
\hline Extrapulmonary TB & $42(41.6)$ & $58(36.0)$ & & & \\
\hline History of previous anti-TB treatment & & & 0.742 & & \\
\hline New treatment & $91(90.1)$ & $147(90.7)$ & & & \\
\hline Retreatment & $10(9.9)$ & $14(9 \cdot 3)$ & & & \\
\hline Abnormal liver function & $4(4 \cdot 0)$ & $28(17.4)$ & 0.001 & $2.828(1.432-5.582)$ & 0.003 \\
\hline Abnormal renal function & $2(2.0)$ & $8(4 \cdot 9)$ & 0.219 & & \\
\hline \multicolumn{6}{|l|}{ Peripheral blood eosinophil } \\
\hline Base eosinophil count/mL & $100(60-200)$ & $130(75-200)$ & 0.177 & & \\
\hline $\begin{array}{l}\text { Base eosinophil percentage, \% of total white } \\
\text { blood cell count }\end{array}$ & $1.7(0.8-3.0)$ & $2.1(0.9-3.3)$ & 0.429 & & \\
\hline Initial eosinophil count/mL & $300(230-465)$ & $400(300-525)$ & 0.012 & & \\
\hline $\begin{array}{l}\text { Initial eosinophil percentage, \% of total } \\
\text { white blood cell count }\end{array}$ & $5.8(5.3-6.9)$ & $6.7(5.6-8.4)$ & $<0.001$ & & \\
\hline Peak eosinophil count/mL & $400(300-500)$ & $500(400-775)$ & $<0.001$ & & \\
\hline $\begin{array}{l}\text { Peak eosinophil percentage, \% of total white } \\
\text { blood cell count }\end{array}$ & $6.4(5.7-8.0)$ & $8.8(6.7-12.8)$ & $<0.001$ & $1.336(1.189-1.501)$ & $<0.001$ \\
\hline
\end{tabular}

Values are presented as median (interquartile range) or number (\%).

CADR, cutaneous adverse drug reaction; OR, odds ratio; CI, confidence interval; TB, tuberculosis.

philia had poor liver function, prolonged use of corticosteroids, and longer hospitalization, which suggested severe disease. In our study, liver function abnormality was associated with CADRs in patients with eosinophilia during anti-TB medication. This finding was consistent with the previous study and suggested that peripheral eosinophilia associated with anti-T'B medication might be similar to that with other antibiotic treatments. However, the relationship between peripheral eosinophil counts and severity of CADRs has not been fully studied. Interestingly, the initial and peak blood eosinophil counts and eosinophil percentages tended to increase with clinical severity of CADRs caused by the anti-TB drugs in this study. Therefore, if patients using anti-TB medication develop peripheral eosinophilia and CADRs, increasing peripheral eosinophil counts might be a warning sign of severe CADRs, requiring careful monitoring for progression.
Discontinuation of offending drugs owing to CADRs has been reported in about $4 \%$ to $5 \%$ of cases $[4,16]$. In this study, the discontinuation rate was about $10 \%$, which was higher than in previous studies. As the study institution is a tertiary university hospital, more severely ill patients might have been enrolled in this study compared with those in previous studies. All anti-TB drugs are known to have the potential to cause CADRs [1]. In our study, ethambutol was the most common anti-TB drug that caused CADRs, which is in contrast to other studies in which pyrazinamide or isoniazid and rifampin have been reported to be the most common offending drugs $[4,28]$.

This study has several limitations as it is a single-center, retrospective study. First, not all patients with TB and peripheral blood eosinophilia that occurred during treatment were enrolled in this study, and anti-parasite antibody tests that could be used to analyze the caus- 
Table 3. Comparison by severity of cutaneous adverse drug reactions in patients with eosinophilia during anti-tuberculosis treatment

\begin{tabular}{|c|c|c|c|c|c|}
\hline Variable & $\begin{array}{l}\text { No CADRs } \\
(\mathrm{n}=101)\end{array}$ & $\begin{array}{l}\text { Pruritus only } \\
\quad(\mathrm{n}=76)\end{array}$ & $\begin{array}{l}\text { Skin rash } \\
\left(\mathrm{n}=5^{8}\right)\end{array}$ & $\begin{array}{l}\text { Stop medication } \\
(\mathrm{n}=27)\end{array}$ & $p$ value \\
\hline Age, yr & $56(39-71)$ & $70(51-77)$ & $58(40-75)$ & $61(50-70)$ & 0.008 \\
\hline Male sex & $66(65 \cdot 3)$ & $46(60.5)$ & $45(77.6)$ & $18(66.7)$ & 0.213 \\
\hline Type of TB & & & & & 0.759 \\
\hline Pulmonary TB & $59(58.4)$ & $50(65.8)$ & $37(63.8)$ & $16(59 \cdot 3)$ & \\
\hline Extrapulmonary TB & $42(41.6)$ & $26(34.2)$ & $21(36.2)$ & $11(40.7)$ & \\
\hline History of previous anti-TB treatment & & & & & 0.980 \\
\hline New treatment & $91(90.1)$ & $69(90.8)$ & $53(91.4)$ & $25(92.6)$ & \\
\hline Retreatment & $10(9.9)$ & $7(9.2)$ & $5(8.6)$ & $2(7 \cdot 4)$ & \\
\hline \multicolumn{6}{|l|}{ Peripheral blood eosinophil } \\
\hline Base eosinophil count/mL & $100(60-200)$ & $130(100-200)$ & $120(70-200)$ & $200(60-280)$ & 0.285 \\
\hline $\begin{array}{l}\text { Base eosinophil percentage, \% of total } \\
\text { white blood cell count }\end{array}$ & $1.7(0.8-3.0)$ & $2.2(0.9-3.3)$ & $1.7(0.8-3.3)$ & $2.0(0.7-3.3)$ & 0.800 \\
\hline Initial eosinophil count/mL & $300(230-465)$ & $370(300-445)$ & $400(300-600)$ & $500(310-900)$ & $<0.001$ \\
\hline $\begin{array}{l}\text { Initial eosinophil percentage, \% of total } \\
\text { white blood cell count }\end{array}$ & $5.8(5 \cdot 3-6.9)$ & $6.1(5.1-7.8)$ & $7.0(5.7-8.3)$ & $8.0(6.7-12.0)$ & $<0.001$ \\
\hline Peak eosinophil count/mL & $400(300-500)$ & $430(300-600)$ & $500(400-753)$ & $1,300(600-2,600)$ & $<0.001$ \\
\hline $\begin{array}{l}\text { Peak eosinophil percentage, \% of total } \\
\text { white blood cell count }\end{array}$ & $6.4(5.7-8.0)$ & $7.3(6.2--9.8)$ & $9.2(7.3-12.4)$ & $17.4(12.0-27.5)$ & $<0.001$ \\
\hline
\end{tabular}

Values are presented as median (interquartile range) or number (\%).

CADR, cutaneous adverse drug reaction; TB, tuberculosis.

Table 4. Offending drugs and reasons for discontinuation of anti-TB treatment

\begin{tabular}{lc}
\hline Variable & Total $(\mathrm{n}=27)$ \\
\hline Reasons for discontinuing anti-TB drugs & \\
Drug rash & $12(44.4)$ \\
Drug fever & $7(25.9)$ \\
DRESS syndrome & $7(25.9)$ \\
\hline SJS/TEN & $1(3.8)$ \\
Offending drugs & \\
\hline Isoniazid & $5(16.7)$ \\
Rifampin & $5(16.7)$ \\
Ethambutol & $10(33.3)$ \\
Pyrazinamide & $6(20.0)$ \\
Unknown & $4(13.3)$ \\
\hline
\end{tabular}

Values are presented as number (\%). There were two offending drugs in three patients (isoniazid and pyrazinamide in one patient, ethambutol and pyrazinamide in two patients). TB, tuberculosis; DRESS, drug reaction with eosinophilia and systemic symptom; SJS/TEN, Stevens-Johnson syndrome/toxic epidermal necrolysis. es of peripheral blood eosinophilia were not evaluated. However, enrolling a larger number of patients, as well as excluding patients with peripheral blood eosinophilia before receipt of anti-TB drugs and previous allergy history, could strengthen the findings of this study. Second, this study did not include a control group. Therefore, no comparison was made between patients with and without eosinophilia.

In conclusion, peripheral blood eosinophilia is common during anti-TB treatment, and the majority of those with eosinophilia experienced CADRs. Higher peripheral blood eosinophilia is associated with more severe CADRs.

\section{KEY MESSAGE}

1. Peripheral blood eosinophilia was relatively common (17.8\%) in 2,234 patients during anti-tuberculosis treatment, with symptoms including itching and a skin rash. 
2. There was a significant relationship between increased peak eosinophil counts and the severity of cutaneous adverse drug reactions

\section{Conflict of interest}

No potential conflict of interest relevant to this article was reported.

\section{Acknowledgments}

This study was supported by the National Research Foundation of Korea funded by the Korean Government (Grant 2016R1DiA1B03931132).

\section{REFERENCES}

1. Rezakovic S, Pastar Z, Kostovic K. Cutaneous adverse drug reactions caused by antituberculosis drugs. Inflamm Allergy Drug Targets 2014;13:241-248.

2. Lehloenya RJ, Dheda K. Cutaneous adverse drug reactions to anti-tuberculosis drugs: state of the art and into the future. Expert Rev Anti Infect Ther 2012;10:475-486.

3. Kolappan C, Subramani R, Kumaraswami V, Santha T, Narayanan PR. Excess mortality and risk factors for mortality among a cohort of TB patients from rural south India. Int J Tuberc Lung Dis 2008;12:81-86.

4. Yee D, Valiquette C, Pelletier M, Parisien I, Rocher I, Menzies D. Incidence of serious side effects from first-line antituberculosis drugs among patients treated for active tuberculosis. Am J Respir Crit Care Med 2003;167:14721477.

5. Surjapranata FJ, Rahaju NN. A case of Stevens-Johnson's syndrome caused by ethambutol. Paediatr Indones 1979;19:195-201.

6. Pegram PS Jr, Mountz JD, O’Bar PR. Ethambutol-induced toxic epidermal necrolysis. Arch Intern Med 1981;141:16771678.

7. Sarkar SK, Purohit SD, Sharma TN, Chawla MP, Gupta DN. Stevens-Johnson syndrome caused by streptomycin. Tubercle 1982;63:137-138.

8. Nunn P, Kibuga D, Gathua S, et al. Cutaneous hypersensitivity reactions due to thiacetazone in HIV-1 seropositive patients treated for tuberculosis. Lancet 1991;337:627-630.

9. Chintu C, Luo C, Bhat G, Raviglione M, DuPont H, Zumla A. Cutaneous hypersensitivity reactions due to thiaceta- zone in the treatment of tuberculosis in Zambian children infected with HIV-I. Arch Dis Child 1993;68:665-668.

10. Akula SK, Aruna AS, Johnson JE, Anderson DS. Cycloserine-induced Stevens-Johnson syndrome in an AIDS patient with multidrug-resistant tuberculosis. Int J Tuberc Lung Dis 1997;1:187-190.

11. Kura MM, Hira SK. Reintroducing antituberculosis therapy after Stevens-Johnson syndrome in human immunodeficiency virus-infected patients with tuberculosis: role of desensitization. Int J Dermatol 2001;40:481-484.

12. Digwood-Lettieri S, Reilly KJ, Haith LR Jr, et al. Levofloxacin-induced toxic epidermal necrolysis in an elderly patient. Pharmacotherapy 2002;22:789-793.

13. Pitche P, Mouzou T, Padonou C, Tchangai-Walla K. Stevens-Johnson syndrome and toxic epidermal necrolysis after intake of rifampicin-isoniazid: report of 8 cases in HIV-infected patients in Togo. Med Trop (Mars) 2005;65:359362.

14. Roufosse F, Weller PF. Practical approach to the patient with hypereosinophilia. J Allergy Clin Immunol 2010;126:39-44.

15. Nutman TB. Evaluation and differential diagnosis of marked, persistent eosinophilia. Immunol Allergy Clin North Am 2007;27:529-549.

16. Blumenthal KG, Youngster I, Rabideau DJ, et al. Peripheral blood eosinophilia and hypersensitivity reactions among patients receiving outpatient parenteral antibiotics. J Allergy Clin Immunol 2015;136:1288-1294.

17. Seung KJ, Bai GH, Kim SJ, Lew WJ, Park SK, Kim JY. The treatment of tuberculosis in South Korea. Int J Tuberc Lung Dis 2003;7:912-919.

18. Park CK, Shin HJ, Kim YI, Lim SC, Yoon JS, Kim YS, et al. Predictors of default from treatment for tuberculosis: a single center case-control study in Korea. J Korean Med Sci 2016;31:254-260.

19. Gotlib J. World Health Organization-defined eosinophilic disorders: 2015 update on diagnosis, risk stratification, and management. Am J Hematol 2015;90:1077-1089.

20. Mockenhaupt M. The current understanding of Stevens-Johnson syndrome and toxic epidermal necrolysis. Expert Rev Clin Immunol 2011;7:803-813.

21. Benichou C. Criteria of drug-induced liver disorders. Report of an international consensus meeting. J Hepatol 1990;11:272-276.

22. Bellomo R, Ronco C, Kellum JA, Mehta RL, Palevsky P; Acute Dialysis Quality Initiative workgroup. Acute renal 
failure: definition, outcome measures, animal models, fluid therapy and information technology needs. The Second International Consensus Conference of the Acute Dialysis Quality Initiative (ADQI) Group. Crit Care 2004;8:R204-R212.

23. Choquet-Kastylevsky G, Intrator L, Chenal C, Bocquet $\mathrm{H}$, Revuz J, Roujeau JC. Increased levels of interleukin 5 are associated with the generation of eosinophilia in drug-induced hypersensitivity syndrome. Br J Dermatol 1998;139:1026-1032.

24. Yawalkar N, Shrikhande M, Hari Y, Nievergelt H, Braathen LR, Pichler WJ. Evidence for a role for IL-5 and eotaxin in activating and recruiting eosinophils in drug-induced cuta- neous eruptions. J Allergy Clin Immunol 2000;106:1171-1176.

25. Gerson D, Sriganeshan V, Alexis JB. Cutaneous drug eruptions: a 5-year experience. J Am Acad Dermatol 2008;59:995999 .

26. Bocquet H, Bagot M, Roujeau JC. Drug-induced pseudolymphoma and drug hypersensitivity syndrome (Drug Rash with Eosinophilia and Systemic Symptoms: DRESS). Semin Cutan Med Surg 1996;15:250-257.

27. Yang J, Yang X, Li M. Peripheral blood eosinophil counts predict the prognosis of drug eruptions. J Investig Allergol Clin Immunol 2013;23:248-255.

28. Roujeau JC, Stern RS. Severe adverse cutaneous reactions to drugs. N Engl J Med 1994;331:1272-1285. 